\title{
Prescribing Patterns for Outpatient Treatment of Constipation, Irritable Bowel Syndrome-Related Constipation, and Opioid-Induced Constipation: A Retrospective Cross-Sectional Study
}

\author{
Katy E. Trinkley, PharmD; Bruce E. Sill, PharmD, MS; Kyle Porter, MAS; \\ and Milap C. Nahata, PharmD, MS
}

\begin{abstract}
BACKGROUND: Despite national recommendations for treatment of constipation, prescribing patterns for treatment are inconsistent, and health care utilization has increased.

OBJECTIVE: To identify patterns in pharmacologic and nonpharmacologic treatment of constipation and associations between treatment and other variables across age groups.

METHODS: This was a retrospective cross-sectional study that used the National Ambulatory Medical Care Survey (NAMCS) to compare prescribing from 2000 to 2004 and from 2005 to 2009. Treatment patterns for constipation, irritable bowel syndrome-related constipation (IBS-C), and opioidinduced constipation were considered.
\end{abstract}

RESULTS: From 2000 to 2009, there were 89.6 million office visits related to constipation: 63.4 million for constipation alone, 28.2 million for IBS-C alone, and 3.7 million for opioid-induced constipation. For constipation, there was an overall decrease in the prescription of combination therapy (17\% vs. $11 \%, P<0.05)$; an increase in the prescription of medication monotherapy (21\% vs. $29 \%, P<0.05)$; decreases in the use of lubricants ( $9 \%$ vs. $2 \%, P<0.05)$ and saline $(7 \%$ vs. $1 \%, P<0.001)$ among patients aged $<18$ years; a decrease in combination therapy ( $31 \%$ vs. $17 \%$, $P<0.05$ ); and age group differences in the prescription of specific medications. For IBS-C and opioid-induced constipation, there were no changes in major treatment category or specific medication. Age, gender, race, ethnicity, payer source, physician specialty, and region were all found to be associated with treatment choice.

CONCLUSIONS: Health care utilization for constipation increased, and prescribing patterns shifted significantly from 2000 to 2009 for constipation and IBS-C. Patterns in treatment were significantly influenced by many factors, including age, gender, and race. Changes in treatment categories over time included a decrease in combination therapy for patients aged $<18$ years and an increase in medication monotherapy for all ages, which are in contrast to national recommendations.

J Manag Care Spec Pharm. 2015;21(11):1077-87

Copyright $\odot 2015$, Academy of Managed Care Pharmacy. All rights reserved.

\section{What is already known about this subject}

A variety of drugs are used for treating constipation.

Prior studies suggest that prescriptions for constipation treatments do not align with expert recommendations.

\section{What this study adds}

Prescribing practices for constipation and irritable bowel syndrome-related constipation (IBS-C) are associated with many factors, including age, gender, and race.

Prescriptions for combination therapy for children and adolescents have decreased over time, while prescriptions for medication monotherapy have increased over time.

Treatment trends for constipation and IBS-C are not aligned with current treatment recommendations.

A lthough constipation is not commonly perceived as a high-risk condition by health care providers, it does commonly and significantly impair quality of life, leading to increased health care utilization and costs across all populations. ${ }^{1,2}$ While the risk of constipation increases with age, $23 \%$ of all children and adolescents suffer from constipation. ${ }^{2-4}$ Because of the widespread incidence of constipation and its impact on quality of life, health care expenditures for constipation average $\$ 1$ billion each year, with additional out-of-pocket expenditures of $\$ 800$ million annually on laxatives alone.,5,

The high cost of constipation management and the impact on quality of life necessitate prompt and appropriate treatment. The treatment goals are to alleviate symptoms (e.g., bloating and abdominal pain) and prevent more severe complications, such as fissures, fecal impaction, hemorrhoids, and loss of stool control. ${ }^{1,7,8}$

Unfortunately, there are limited evidence-based treatment options, and most of the current treatments are based largely on historical practices ${ }^{1,9}$; consequently, there is great variation in the treatments prescribed, varying by factors such as geographical location and physician specialty., ${ }^{2,10-12}$ To standardize and optimize treatments, expert recommendations suggest approaches to treating the different types of constipation., ${ }^{1,9-10}$ For chronic constipation, the recommendations for adults and children are to start with nonpharmacologic lifestyle modifications that include increased exercise, water, and fiber intake. The first step for children also includes the addition of medications, whereas for adults, medications are not added until the second step. ${ }^{7,9,12}$ Treatment of irritable bowel syndrome-related 


\section{Prescribing Patterns for Outpatient Treatment of Constipation, Irritable Bowel Syndrome-Related Constipation, and Opioid-Induced Constipation: A Retrospective Cross-Sectional Study}

constipation (IBS-C) begins with increasing fiber and then adding medications. ${ }^{1}$ Since 2009, an updated set of clinical practice guidelines has been published; however, the general recommendations for treatment have not changed.

Examining the changes in the prescription of treatment for constipation from 1997 to 2006, Trinkley et al. (2010) found that the use of nonpharmacologic monotherapy decreased and the use of medications alone increased. ${ }^{12}$ However, this study did not consider IBS-C nor evaluate the treatment of opioidinduced constipation, which are common forms of constipation. To date, no studies have evaluated the prescribing patterns for IBS-C or opioid-induced constipation nor have studies considered the influence of new drugs on prescribing patterns for constipation.

Understanding the prescribing patterns for constipation and IBS-C treatment and how they align with guidelines is of particular importance to managed care pharmacy. Identification of prescribing patterns that deviate from evidence-based recommendations and standards allow for targeted interventions that are meant to improve patient outcomes and reduce health care utilization and costs. To that end, the purpose of this study was to identify the patterns in the medication management of constipation and the associations between treatment and other variables. This study was deemed exempt by the institutional review board for The Ohio State University.

\section{Methods}

\section{The National Ambulatory Medical Care Survey}

The National Ambulatory Medical Care Survey (NAMCS) provides data on outpatient physician office visits in the United States, such as patient symptoms, diagnoses, medications, and demographics. ${ }^{13}$ Since 1973, and annually since 1989, data have been collected by the National Center for Health Statistics and the Centers for Disease Control and Prevention for the purpose of providing objective, reliable information on the provision of medical care. Randomly selected U.S. physicians voluntarily participate and are trained for standardization prior to recording and submitting survey data. Of these physicians, only a randomly selected subset in a given time frame is included in NAMCS to account for confounding variables and for external validity. The subset is defined by a multistage design composed of 3 nested stages: geographical location, physician specialty (of the geographical location), and patient visits (of the physician specialty). Physicians complete data collection for patient visits over a l-week period. The data are anonymous and publicly available online.

\section{Study Design}

This was a retrospective cross-sectional study using NAMCS data from 2000 to 2009 (the most recent data available) on the differences in time periods in pharmacologic and nonpharmacologic treatments for constipation across different age groups.

\begin{tabular}{|c|c|c|c|c|}
\hline Visit Type ${ }^{a}$ & All & $\begin{array}{c}\text { All-Cause } \\
\text { Constipation } \\
\text { (No IBS-C) } \\
\end{array}$ & $\begin{array}{l}\text { IBS-C } \\
\text { Alone }\end{array}$ & $\begin{array}{l}\text { Opioid- } \\
\text { Induced } \\
\text { Constipation } \\
\text { Alone }\end{array}$ \\
\hline Visits (n, millions) & 89.6 & 63.4 & 28.2 & 3.7 \\
\hline Female & 69 & 65 & 80 & 68 \\
\hline \multicolumn{5}{|l|}{ Race } \\
\hline Caucasian & 85 & 83 & 89 & 81 \\
\hline African American & 10 & 12 & 8 & 12 \\
\hline Asian/Pacific Islander & 3 & 3 & 3 & 5 \\
\hline Other & 2 & 2 & 0 & 2 \\
\hline \multicolumn{5}{|l|}{ Ethnicity } \\
\hline Non-Hispanic & 81 & 80 & 84 & 85 \\
\hline \multicolumn{5}{|l|}{ Payment source } \\
\hline Private insurance & 51 & 46 & 61 & 29 \\
\hline Medicaid/Medicare & 40 & 45 & 30 & 62 \\
\hline Self-pay & 3 & 3 & 4 & 5 \\
\hline Other/unknown & 6 & 6 & 6 & 4 \\
\hline \multicolumn{5}{|l|}{ Physician specialty } \\
\hline Gastroenterology $\mathrm{b}$ & 19 & 14 & 23 & 11 \\
\hline General/family practice & 26 & 25 & 29 & 46 \\
\hline Internal medicine & 20 & 17 & 28 & 18 \\
\hline Pediatrics & 15 & 24 & 2 & - \\
\hline General surgery & 2 & & 1 & 2 \\
\hline OBGYN & 4 & & 3 & 2 \\
\hline Other specialty & 14 & 20 & 12 & 22 \\
\hline \multicolumn{5}{|l|}{ Region } \\
\hline Northeast & 16 & 14 & & \\
\hline Midwest & 19 & 18 & & \\
\hline South & 45 & 48 & & \\
\hline West & 20 & 20 & & \\
\hline \multicolumn{5}{|c|}{$\begin{array}{l}{ }^{a} \text { All values are percentages except first row, which is weighted frequency. } \\
{ }^{b} \text { Not available for } 2008-2009 \text {. } \\
\text { IBS-C = irritable bowel syndrome-related constipation; OBGYN= obstetrics and } \\
\text { gynecology. }\end{array}$} \\
\hline
\end{tabular}

The NAMCS database contains data on patient-specific office visits, including constipation-related visits.

Data from the NAMCS database were limited to office visits for patients that were associated with an International Classification of Diseases, Ninth Revision, Clinical Modification (ICD-9-CM) code related to constipation or with constipation recorded as the "reason for visit" (Appendix A, available in online article). Visits related to constipation included allcause constipation, IBS-C, and opioid-induced constipation. Since there is no ICD-9-CM code for IBS- $C$, the nonspecific ICD-9-CM code for IBS was used. It was assumed that a constipation treatment would not be prescribed to someone with diarrhea-predominant IBS (IBS-D). This definition of IBS-D is limited in that some patients with IBS-D may be prescribed fiber; however, this is the best definition available using 
TABLE 2 Treatment Differences in Time Periods by Major Treatment Category, 2000-2009, Percentage of Constipation and/or IBS Visits (Range)

\begin{tabular}{|c|c|c|c|c|c|c|c|c|c|c|}
\hline \multirow{3}{*}{$\begin{array}{l}\text { Age Range, Years } \\
<18\end{array}$} & \multirow{3}{*}{\begin{tabular}{|c|}
$\begin{array}{c}\text { Weighted Number of } \\
\text { Visits } \\
\text { (Millions) }\end{array}$ \\
1.2
\end{tabular}} & \multirow{2}{*}{$\frac{\text { Time Period }}{2000-2004}$} & \multicolumn{2}{|c|}{$\begin{array}{c}\text { Medication and } \\
\text { Nonpharmacologic } \\
\text { Therapy }^{\mathrm{a}}\end{array}$} & \multicolumn{2}{|c|}{ Medication Only } & \multicolumn{2}{|c|}{$\begin{array}{c}\text { Nonpharmacologic } \\
\text { Therapy Only }\end{array}$} & \multicolumn{2}{|c|}{ No Therapy } \\
\hline & & & 29 & $(21-39)^{b}$ & 20 & $(12-32)$ & 20 & $(13-28)$ & 31 & $(24-39)$ \\
\hline & & 2005-2009 & 16 & $(10-24)^{b}$ & 27 & $(20-36)$ & 19 & $(14-26)$ & 38 & $(31-45)$ \\
\hline \multirow{2}{*}{$18-44$} & \multirow{2}{*}{9.4} & $2000-2004$ & 11 & $(7-18)$ & 17 & $(11-24)$ & 28 & $(21-36)$ & 45 & $(37-52)$ \\
\hline & & 2005-2009 & 9 & $(5-16)$ & 21 & $(15-29)$ & 22 & $(16-30)$ & 47 & $(38-56)$ \\
\hline \multirow{2}{*}{$45-64$} & \multirow{2}{*}{10.5} & $2000-2004$ & 10 & $(6-17)$ & 6 & $(4-10)^{c}$ & 20 & $(14-28)$ & 64 & $(55-72)$ \\
\hline & & $2005-2009$ & 8 & $(5-12)$ & 20 & $(14-26)^{c}$ & 18 & $(12-26)$ & 55 & $(46-62)$ \\
\hline \multirow{2}{*}{$\geq 65$} & \multirow{2}{*}{2.8} & $2000-2004$ & 6 & $(3-10)$ & 19 & $(14-26)$ & 24 & $(18-31)$ & 51 & $(43-59)$ \\
\hline & & 2005-2009 & 7 & $(4-12)$ & 24 & $(19-31)$ & 21 & $(16-29)$ & 47 & $(41-54)$ \\
\hline \multirow{2}{*}{ All visits } & \multirow{2}{*}{28.2} & $2000-2004$ & 13 & $(10-17)$ & 15 & $(12-19)^{\mathrm{d}}$ & 23 & $(20-27)$ & 48 & $(44-53)$ \\
\hline & & 2005-2009 & 10 & $(8-12)$ & 23 & $(20-27)^{d}$ & 20 & $(17-24)$ & 47 & $(43-51)$ \\
\hline
\end{tabular}

a Represents patients prescribed at least 1 medication.

${ }^{b} \mathrm{P}<0.05$ for 2000-2004 versus 2005-2009.

${ }^{c} P<0.001$.

$d P<0.01$.

IBS = irritable bowel syndrome.

current coding systems. There is also no ICD-9-CM code for opioid-induced constipation, so it was defined as patients with an ICD-9-CM code for constipation and concurrent opioid use. This definition for opioid-induced constipation was chosen because it is recommended that patients taking opioids for any period of time should be treated either prophylactically or acutely with certain constipation medications, regardless of preexisting constipation. ${ }^{14}$ All-cause constipation included patients with opioid-induced constipation, given that the study definition of opioid-induced constipation included an ICD9-CM code for constipation.

Patients and visits were not linked in the NAMCS database. Data collection in NAMCS occurs over 1 week for each participating provider, so the possibility of capturing data for a given patient more than once is not likely. All constipation medications (over-the-counter and prescription) that were new, administered, or continued at that visit were collected and identified by NAMCS medication codes (Appendix B, available in online article). Data on nonpharmacologic treatments for constipation were also collected, including diet and behavior changes. Diet and behavior changes are predefined options on the NAMCS data collection form. The presence of comorbidities with potential to affect constipation symptoms, or alter treatment, were collected and identified by diagnosis codes. Similarly, concurrent medications (e.g., opioids and anticholinergics) with potential to affect constipation symptoms or alter treatment were collected by NAMCS unique drug codes. Demographic and other variables that may influence constipation management were considered and included age, sex, race, comorbidities, concurrent medications, geographical location, tobacco use, physician specialty, insurance type, pharma- cologic and nonpharmacologic treatments, and procedures (Appendix C, available in online article).

\section{Outcome Measures}

The primary outcome was the proportion of office visits in which each therapy was prescribed for (a) all constipation and IBS-C visits, including opioid-induced; (b) all-cause constipation, including opioid-induced; (c) IBS-C alone; and (d) opioidinduced constipation alone. These proportions were estimated for specific medications, therapeutic classes, and medication and/or nonpharmacologic treatment in general. Estimates were obtained overall, by year, and by diagnosis (the composite of all visits, all-cause constipation, IBS-C alone, and opioidinduced constipation alone). Prescribing activity for each visit was the outcome measure for assessing association of patient and physician characteristics with therapy selection. Data were analyzed, and results were reported as the proportion of prescribed treatments by diagnosis, concurrent medications, comorbidities, treatment type, therapeutic class, new or continuing therapy, physician specialty, and other demographics. Also, an assessment of differences in time periods and predictors of prescribing were reported.

\section{Statistical Analyses}

All analyses used NAMCS sampling weights. In order to obtain correct standard error estimates, all patient visits from 2000 to 2009 were used in the analyses. Subgroup analyses were then used to obtain results for the desired target populations of constipation-related visits. Separate analyses were performed for each combination of age group (less than 18, $18-44,45-64$, and $\geq 65$ ) and type of constipation (all-cause 


\section{Prescribing Patterns for Outpatient Treatment of Constipation, Irritable Bowel Syndrome-Related Constipation, and Opioid-Induced Constipation: A Retrospective Cross-Sectional Study}

TABLE 3 Treatment Differences in Time Periods by Medication Type, 2000-2009, Percentage of Constipation and/or IBS Visits with Prescribed Medication (Range)

\begin{tabular}{|c|c|c|c|c|c|c|c|c|c|c|c|c|c|}
\hline 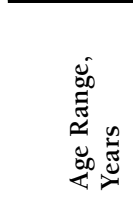 & 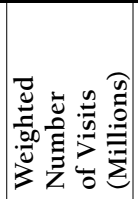 & 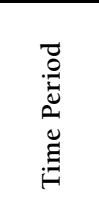 & 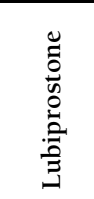 & 节 & 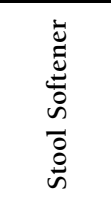 & 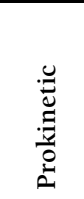 & 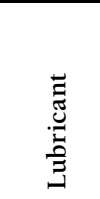 & 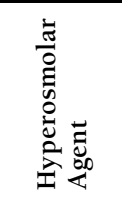 & 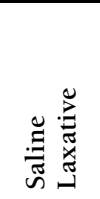 & 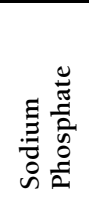 & 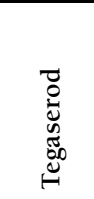 & 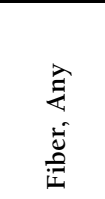 & 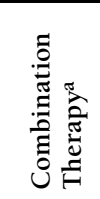 \\
\hline \multirow[b]{2}{*}{$<18$} & \multirow{2}{*}{1.2} & $\begin{array}{l}2000- \\
2004\end{array}$ & - & $\begin{array}{c}10 \\
(4-23)\end{array}$ & $\begin{array}{c}6 \\
(2-15) \\
\end{array}$ & $\begin{array}{c}2 \\
(1-8) \\
\end{array}$ & $\begin{array}{c}17 \\
(9-32)^{\mathrm{b}}\end{array}$ & $\begin{array}{c}74 \\
(58-85)^{c} \\
\end{array}$ & $\begin{array}{c}6 \\
(2-17)^{\mathrm{b}} \\
\end{array}$ & - & - & $\begin{array}{c}3 \\
(1-13) \\
\end{array}$ & $\begin{array}{c}17 \\
(8-31)\end{array}$ \\
\hline & & $\begin{array}{l}2005- \\
2009\end{array}$ & - & $\begin{array}{c}8 \\
(4-17)\end{array}$ & $\begin{array}{c}3 \\
(1-8)\end{array}$ & - & $\begin{array}{c}2 \\
(0-7)^{\mathrm{b}}\end{array}$ & $\begin{array}{c}90 \\
(82-95)^{c}\end{array}$ & $\begin{array}{c}0 \\
(0-1)^{\mathrm{b}}\end{array}$ & - & - & $\begin{array}{c}3 \\
(1-9) \\
\end{array}$ & $\begin{array}{c}6 \\
(2-14)\end{array}$ \\
\hline \multirow{2}{*}{$18-44$} & \multirow{2}{*}{9.4} & $\begin{array}{l}2000- \\
2004\end{array}$ & - & $\begin{array}{c}3 \\
(1-11)^{c}\end{array}$ & $\begin{array}{c}21 \\
(9-42)\end{array}$ & $\begin{array}{c}4 \\
(1-24)\end{array}$ & $\begin{array}{c}2 \\
(0-8)\end{array}$ & $\begin{array}{c}34 \\
(19-53)\end{array}$ & $\begin{array}{c}3 \\
(1-12)\end{array}$ & $\begin{array}{c}0 \\
(0-2)\end{array}$ & $\begin{array}{c}22 \\
(9-42)\end{array}$ & $\begin{array}{c}25 \\
(14-40)\end{array}$ & $\begin{array}{c}15 \\
(5-38)\end{array}$ \\
\hline & & $\begin{array}{l}2005- \\
2009\end{array}$ & $\begin{array}{c}9 \\
(3-21)\end{array}$ & $\begin{array}{c}15 \\
(7-28)^{c}\end{array}$ & $\begin{array}{c}13 \\
(6-27)\end{array}$ & $\begin{array}{c}6 \\
(1-23)\end{array}$ & $\begin{array}{c}4 \\
(1-23)\end{array}$ & $\begin{array}{c}36 \\
(23-51)\end{array}$ & $\begin{array}{c}0 \\
(0-2)\end{array}$ & - & $\begin{array}{c}8 \\
(2-30)\end{array}$ & $\begin{array}{c}24 \\
(12-41)\end{array}$ & $\begin{array}{c}19 \\
(10-33)\end{array}$ \\
\hline \multirow{2}{*}{$45-64$} & \multirow{2}{*}{10.5} & $\begin{array}{l}2000- \\
2004\end{array}$ & - & $\begin{array}{c}13 \\
(4-32)^{c}\end{array}$ & $\begin{array}{c}9 \\
(3-24)\end{array}$ & $\begin{array}{c}9 \\
(3-24)\end{array}$ & - & $\begin{array}{c}44 \\
(30-60)\end{array}$ & $\begin{array}{c}6 \\
(1-19)\end{array}$ & - & $\begin{array}{c}11 \\
(4-28)\end{array}$ & $\begin{array}{c}14 \\
(6-30)\end{array}$ & $\begin{array}{c}4 \\
(1-19)\end{array}$ \\
\hline & & $\begin{array}{l}2005- \\
2009\end{array}$ & $\begin{array}{c}6 \\
(2-17) \\
\end{array}$ & $\begin{array}{c}1 \\
(0-5)^{c}\end{array}$ & $\begin{array}{c}8 \\
(3-16)\end{array}$ & $\begin{array}{c}3 \\
(1-14)\end{array}$ & - & $\begin{array}{c}55 \\
(39-69)\end{array}$ & $\begin{array}{c}1 \\
(0-4)\end{array}$ & - & $\begin{array}{c}13 \\
(6-26) \\
\end{array}$ & $\begin{array}{c}15 \\
(7-29) \\
\end{array}$ & $\begin{array}{c}4 \\
(1-10)\end{array}$ \\
\hline \multirow[t]{2}{*}{$\geq 65$} & \multirow{2}{*}{2.8} & $\begin{array}{l}2000- \\
2004\end{array}$ & - & $\begin{array}{c}9 \\
(3-20)\end{array}$ & $\begin{array}{c}20 \\
(10-37)\end{array}$ & $\begin{array}{c}2 \\
(1-8) \\
\end{array}$ & $\begin{array}{c}3 \\
(1-12) \\
\end{array}$ & $\begin{array}{c}39 \\
(24-56) \\
\end{array}$ & $\begin{array}{c}10 \\
(4-22)^{c}\end{array}$ & $\begin{array}{c}3 \\
(1-13)\end{array}$ & $\begin{array}{c}14 \\
(5-30)^{c}\end{array}$ & $\begin{array}{c}23 \\
(13-38) \\
\end{array}$ & $\begin{array}{c}23 \\
(12-37) \\
\end{array}$ \\
\hline & & $\begin{array}{l}2005- \\
2009\end{array}$ & $\begin{array}{c}6 \\
(2-15)\end{array}$ & $\begin{array}{c}8 \\
(4-16)\end{array}$ & $\begin{array}{c}21 \\
(12-33)\end{array}$ & $\begin{array}{c}4 \\
(1-15)\end{array}$ & $\begin{array}{c}0 \\
(0-2)\end{array}$ & $\begin{array}{c}46 \\
(34-58)\end{array}$ & $\begin{array}{c}2 \\
(0-7)^{c}\end{array}$ & - & $\begin{array}{c}2 \\
(1-8)^{c}\end{array}$ & $\begin{array}{c}29 \\
(19-43)\end{array}$ & $\begin{array}{c}18 \\
(10-29)\end{array}$ \\
\hline \multirow{2}{*}{ All visits } & \multirow{2}{*}{28.2} & $\begin{array}{l}2000- \\
2004\end{array}$ & - & $\begin{array}{c}8 \\
(5-14)\end{array}$ & $\begin{array}{c}13 \\
(8-21)\end{array}$ & $\begin{array}{c}3 \\
(1-9)\end{array}$ & $\begin{array}{c}8 \\
(4-13)^{b}\end{array}$ & $\begin{array}{c}52 \\
(42-61)\end{array}$ & $\begin{array}{c}6 \\
(3-11)^{b}\end{array}$ & $\begin{array}{c}1 \\
(0-3)\end{array}$ & $\begin{array}{c}10 \\
(6-17)\end{array}$ & $\begin{array}{c}15 \\
(10-21)\end{array}$ & $\begin{array}{c}16 \\
(10-24)\end{array}$ \\
\hline & & $\begin{array}{l}2005- \\
2009\end{array}$ & $\begin{array}{c}5 \\
(3-9)\end{array}$ & $\begin{array}{c}8 \\
(5-12)\end{array}$ & $\begin{array}{c}11 \\
(8-16)\end{array}$ & $\begin{array}{c}3 \\
(1-8)\end{array}$ & $\begin{array}{c}2 \\
(0-5)^{\mathrm{b}}\end{array}$ & $\begin{array}{c}58 \\
(50-65)\end{array}$ & $\begin{array}{c}1 \\
(0-2)^{b}\end{array}$ & - & $\begin{array}{c}5 \\
(3-10)\end{array}$ & $\begin{array}{c}18 \\
(13-25)\end{array}$ & $\begin{array}{c}12 \\
(8-17)\end{array}$ \\
\hline
\end{tabular}

Note: empty cells indicate zero occurances in the sample

aRepresents patients prescribed at least 1 medication and nonpharmacologic treatment.

${ }^{b} P<0.01$.

${ }^{c} P<0.05$ for 2000-2004 versus 2005-2009.

IBS = irritable bowel syndrome.

constipation, IBS-C, opioid-induced constipation). Descriptive statistics for patient and physician characteristics were calculated as percentages of visits with constipation or IBS-C diagnosis or reason for visit. Prescribing differences in time periods were estimated for major treatment and drug classes. The study period was grouped into two 5 -year periods, and differences between time periods were tested by weighted logistic regression models. Periods of 5 years were chosen to ensure sufficient raw (unweighted) data counts. Weighted logistic regression models were also used to test for associations of patient and physician characteristics with treatments. In the case of 2 or more medications prescribed for a single visit, each medication was considered independently. Indicators for the 5-year time period (2000-2004 or 2005-2009) were included as control variables in each model. Independent variables were gender, race, ethnicity, payment source, physician specialty, and geographical region of physician practice. The 4 major treatment categories (medication, nonpharmacologic therapy, both, and neither) were dependent variables in separate models. For each outcome, a model was fit using all visits, and separate models were fit to each of 4 age groups. After modeling associations for these 4 treatment categories, weighted logistic regression models were fit using the subset of visits from eligible patients who were receiving medication for the treatment of constipation symptoms. The purpose of using these models was to determine factors associated with the choice of specific medications. An alpha level of 0.05 was used as the level of significance for all analyses. All analyses were conducted using STATA statistical software version 10.1 (StataCorp, College Station, TX).

\section{Results}

\section{All Constipation and IBS-C Visits}

There were 89.6 million visits for constipation and IBS-C between 2000 and 2009 (Table 1). The only major change in overall treatment category or specific medication prescribed was an increase in medication monotherapy over time $(P<0.01$; Tables 2 and 3).

\section{All-Cause Constipation}

As shown in Table 4, 63.4 million visits for constipation that were not IBS-related were identified. Across all visits, there were significant changes in the major treatment category. 
TABLE 4 Treatment Differences in Time Periods by Major Treatment Category, 2000-2009, Percentage of All-Cause Constipation Visits (Range)

\begin{tabular}{|c|c|c|c|c|c|c|c|c|c|c|}
\hline \multirow{3}{*}{$\begin{array}{l}\text { Age Range, Years } \\
<18\end{array}$} & \multirow{3}{*}{$\begin{array}{c}\begin{array}{c}\text { Weighted Number } \\
\text { of Visits } \\
\text { (Millions) }\end{array} \\
18.0\end{array}$} & \multirow{2}{*}{$\frac{\text { Time Period }}{2000-2004}$} & \multicolumn{2}{|c|}{$\begin{array}{c}\text { Medication and } \\
\text { Nonpharmacologic } \\
\text { Therapy }^{\mathrm{a}}\end{array}$} & \multicolumn{2}{|c|}{ Medication Only } & \multicolumn{2}{|c|}{$\begin{array}{c}\text { Nonpharmacologic } \\
\text { Therapy Only }\end{array}$} & \multicolumn{2}{|c|}{ No Therapy } \\
\hline & & & 31 & $(22-41)^{b}$ & 21 & $(13-28)$ & 19 & $(13-28)$ & 28 & $(21-37)$ \\
\hline & & $2005-2009$ & 17 & $(11-25)^{b}$ & 28 & $(21-37)$ & 19 & $(13-26)$ & 36 & $(29-44)$ \\
\hline \multirow{2}{*}{$18-44$} & \multirow{2}{*}{11.9} & $2000-2004$ & 15 & $(8-25)$ & 31 & $(20-45)$ & 19 & $(11-30)$ & 36 & $(26-46)$ \\
\hline & & $2005-2009$ & 12 & $(6-20)$ & 28 & $(20-39)$ & 26 & $(18-36)$ & 34 & $(25-45)$ \\
\hline \multirow{2}{*}{$45-64$} & \multirow{2}{*}{13.3} & $2000-2004$ & 14 & $(8-25)$ & 8 & $(4-15) c$ & 16 & $(8-29)$ & 62 & $(49-74)$ \\
\hline & & $2005-2009$ & 11 & $(7-18)$ & 26 & $(18-35)^{c}$ & 15 & $(10-23)$ & 48 & $(38-58)$ \\
\hline \multirow{2}{*}{$\geq 65$} & \multirow{2}{*}{20.3} & $2000-2004$ & 5 & $(3-10)$ & 22 & $(15-31)$ & 26 & $(18-34)$ & 48 & $(39-57)$ \\
\hline & & $2005-2009$ & 7 & $(4-13)$ & 31 & $(24-39)$ & 20 & $(13-28)$ & 42 & $(35-49)$ \\
\hline \multirow{2}{*}{ All visits } & \multirow{2}{*}{63.4} & $2000-2004$ & 17 & $(13-21)^{b}$ & 21 & $(16-26)^{b}$ & 21 & $(17-25)$ & 42 & $(37-48)$ \\
\hline & & $2005-2009$ & 11 & $(9-15)^{b}$ & 29 & $(24-33)^{b}$ & 20 & $(16-24)$ & 40 & $(36-45)$ \\
\hline
\end{tabular}

aRepresents patients prescribed at least 1 medication.

${ }^{b} P<0.05$ for 2000-2004 versus 2005-2009.

${ }^{c} P<0.01$.

Across all Age Groups. Only age and gender were associated with choice of major treatment category across all groups. Adults aged 65 years and older were less likely to receive medication and nonpharmacologic therapy than any other age group (aged $<18$ years: odds ratio $[\mathrm{OR}]=0.25,95 \%$ confidence interval $[C I]=0.13-0.46, P<0.001$; aged $18-44$ years: $O R=0.46$, $95 \% \mathrm{CI}=0.22-0.96, P=0.04$; aged $45-65$ years: $\mathrm{OR}=0.46,95 \%$ $\mathrm{CI}=0.24-0.90, P=0.02$ ). Males were more likely to receive medications and nonpharmacologic therapy $(\mathrm{OR}=1.6,95 \%$ $\mathrm{CI}=1.0-2.3, P=0.03)$ and less likely to receive nonpharmacologic therapy only $(\mathrm{OR}=0.66,95 \% \mathrm{CI}=0.48-0.92, \mathrm{P}=0.01)$.

Across all age groups, the choice of specific medication was associated with age and race. Adults had lower odds of receiving hyperosmolar agents than children (aged 18-44 years: $\mathrm{OR}=0.14,95 \% \mathrm{CI}=0.06-0.32, P<0.001$; aged $45-64$ years: $\mathrm{OR}=0.29,95 \% \mathrm{CI}=0.13-0.67, P=0.004$; aged $\geq 65$ years: $\mathrm{OR}=0.19,95 \% \mathrm{CI}=0.08-0.46, P<0.001$; Table 5). AfricanAmerican patients were more likely to receive prokinetics than Caucasian patients $(\mathrm{OR}=6.7,95 \% \mathrm{CI}=1.4-31.6, \mathrm{P}=0.02)$.

By Age Group. There were associations between major treatment category and ethnicity, physician specialty, and region by age group. Hispanic patients had higher odds than nonHispanic patients of receiving only nonpharmacologic treatment in patients aged $18-44$ years $(\mathrm{OR}=4.4,95 \% \mathrm{CI}=1.6-12.3$, $P=0.01$ ), but lower odds than non-Hispanic patients of receiving only nonpharmacologic therapy in patients aged 45-64 years $(\mathrm{OR}=0.14,95 \% \mathrm{CI}=0.03-0.70, \mathrm{P}=0.02)$. For patients younger than 18 years, pediatricians were more likely to prescribe medication and nonpharmacologic therapy than family medicine practitioners $(\mathrm{OR}=6.9,95 \% \mathrm{CI}=2.0-24.5, \mathrm{P}=0.003)$. No therapy was prescribed more often in the South than in the Midwest $(\mathrm{OR}=2.3,95 \% \mathrm{CI}=1.1-4.5, \quad P=0.02)$ or West
$(\mathrm{OR}=2.3,95 \% \mathrm{CI}=1.2-4.4, P=0.02)$. In elderly patients, family medicine practitioners were more likely to prescribe stool softeners than gastroenterologists $(\mathrm{OR}=10.2,95 \% \mathrm{CI}=2.3-44.6$, $P=0.002)$.

\section{IBS-C Alone}

A total of 28.2 million visits for IBS were identified. Across all visits, there were no significant changes in the prescription of major treatment categories or specific agents (Table 6). However, when examining prescribing patterns by patient age, there were significant changes in prescribing within the age groups. The prescription of nonpharmacologic treatments decreased for patients aged $18-44$ years $(P<0.05)$, tegaserod use increased in patients aged $45-64$ years $(P<0.05)$, and combination treatments increased in patients aged $45-64$ years $(P<0.05$; Tables 6 and 7$)$.

Across all IBS-C visits, there were associations between major treatment category and payment source. Patient visits covered primarily by private insurance were more likely to include medication and nonpharmacologic treatments, compared with those covered primarily by Medicare or Medicaid $(\mathrm{OR}=13.2,95 \% \mathrm{CI}=1.6-107.9, P=0.02)$.

Across All Age Groups. Choice of specific medication was associated with gender across all age groups. Females had higher odds of being prescribed tegaserod $(\mathrm{OR}=14,95 \%$ $\mathrm{CI}=2-87, P=0.004)$ and lower odds of being prescribed fiber $(\mathrm{OR}=0.11,95 \% \mathrm{CI}=0.02-0.81, P=0.03)$ than males.

By Age Group. There were associations between major treatment category and gender, race, pay source, and physician specialty by age group. In patients aged 45-64 years, males had higher odds than females of receiving nonpharmacologic treatment only $(\mathrm{OR}=2.7,95 \% \mathrm{CI}=1.1-6.7, P=0.03)$. In patients aged 18-44 years, family practitioners prescribed 


\section{Prescribing Patterns for Outpatient Treatment of Constipation, Irritable Bowel Syndrome-Related Constipation, and Opioid-Induced Constipation: A Retrospective Cross-Sectional Study}

TABLE 5 Treatment Differences in Time Periods by Medication Type, 2000-2009, Percentage of All-Cause Constipation Visits with Prescribed Medication (Range)

\begin{tabular}{|c|c|c|c|c|c|c|c|c|c|c|c|c|c|}
\hline 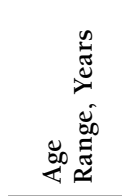 & 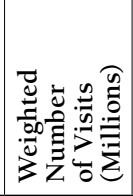 & 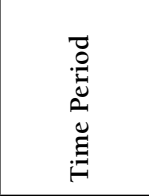 & 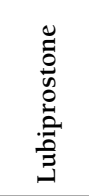 & 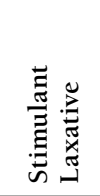 & 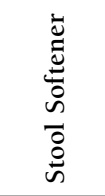 & 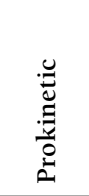 & 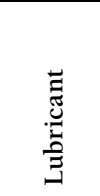 & 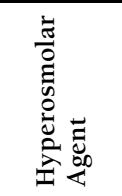 & 节 & 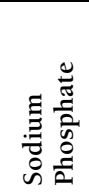 & 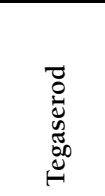 & $\begin{array}{l}\widehat{E} \\
\hat{\mathrm{E}} \\
\hat{\mathrm{E}}\end{array}$ & 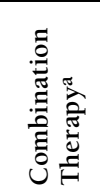 \\
\hline \multirow{2}{*}{$<18$} & \multirow{2}{*}{18.0} & $2000-2004$ & - & $\begin{array}{c}10 \\
(4-23) \\
\end{array}$ & $\begin{array}{c}6 \\
(2-15) \\
\end{array}$ & $\begin{array}{c}2 \\
(1-8)\end{array}$ & $\begin{array}{c}17 \\
(9-32)^{\mathrm{b}}\end{array}$ & $\begin{array}{c}74 \\
(58-85)^{c}\end{array}$ & $\begin{array}{c}6 \\
(2-17)^{b} \\
\end{array}$ & - & - & $\begin{array}{c}3 \\
(1-13)\end{array}$ & $\begin{array}{c}17 \\
(8-31) \\
\end{array}$ \\
\hline & & 2005-2009 & - & $\begin{array}{c}8 \\
(4-18) \\
\end{array}$ & $\begin{array}{c}3 \\
(1-8)\end{array}$ & - & $\begin{array}{c}2 \\
(0-7)^{b}\end{array}$ & $\begin{array}{c}90 \\
(82-95)^{c} \\
\end{array}$ & $\begin{array}{c}0 \\
(0-1)^{\mathrm{b}}\end{array}$ & - & - & $\begin{array}{c}3 \\
(1-9) \\
\end{array}$ & $\begin{array}{c}6 \\
(2-14)\end{array}$ \\
\hline \multirow{2}{*}{$18-44$} & \multirow{2}{*}{11.9} & $2000-2004$ & - & $\begin{array}{c}3 \\
(1-14)^{c} \\
\end{array}$ & $\begin{array}{c}26 \\
(12-49) \\
\end{array}$ & $\begin{array}{c}3 \\
(0-16) \\
\end{array}$ & $\begin{array}{c}3 \\
(1-10) \\
\end{array}$ & $\begin{array}{c}38 \\
(20-59) \\
\end{array}$ & $\begin{array}{c}4 \\
(1-14) \\
\end{array}$ & $\begin{array}{c}0 \\
(0-2) \\
\end{array}$ & $\begin{array}{c}24 \\
(10-48) \\
\end{array}$ & $\begin{array}{c}17 \\
(8-33) \\
\end{array}$ & $\begin{array}{c}18 \\
(6-44) \\
\end{array}$ \\
\hline & & 2005-2009 & $\begin{array}{c}5 \\
(1-16)\end{array}$ & $\begin{array}{c}17 \\
(9-32)^{c}\end{array}$ & $\begin{array}{c}15 \\
(7-31)\end{array}$ & $\begin{array}{c}5 \\
(1-28) \\
\end{array}$ & $\begin{array}{c}5 \\
(1-26) \\
\end{array}$ & $\begin{array}{c}41 \\
(27-57)\end{array}$ & $\begin{array}{c}0 \\
(0-3)\end{array}$ & - & $\begin{array}{c}8 \\
(1-35) \\
\end{array}$ & $\begin{array}{c}21 \\
(9-40) \\
\end{array}$ & $\begin{array}{c}22 \\
(12-37)\end{array}$ \\
\hline \multirow{2}{*}{$45-64$} & \multirow{2}{*}{13.3} & $2000-2004$ & - & $\begin{array}{c}15 \\
(4-41) c\end{array}$ & $\begin{array}{c}8 \\
(2-26) \\
\end{array}$ & $\begin{array}{c}6 \\
(1-27)\end{array}$ & - & $\begin{array}{c}55 \\
(35-73) \\
\end{array}$ & $\begin{array}{c}8 \\
(2-27) c \\
\end{array}$ & - & $\begin{array}{c}12 \\
(3-35)\end{array}$ & $\begin{array}{c}3 \\
(1-12) \\
\end{array}$ & $\begin{array}{c}6 \\
(1-27)\end{array}$ \\
\hline & & 2005-2009 & $\begin{array}{c}7 \\
(2-20)\end{array}$ & $\begin{array}{c}1 \\
(0-6)^{c}\end{array}$ & $\begin{array}{c}9 \\
(4-19)\end{array}$ & - & - & $\begin{array}{c}59 \\
(42-74) \\
\end{array}$ & $\begin{array}{c}1 \\
(0-5)^{c}\end{array}$ & - & $\begin{array}{c}11 \\
(4-24) \\
\end{array}$ & $\begin{array}{c}15 \\
(6-31) \\
\end{array}$ & $\begin{array}{c}4 \\
(1-12) \\
\end{array}$ \\
\hline \multirow{2}{*}{$\geq 65$} & \multirow{2}{*}{20.3} & $2000-2004$ & - & $\begin{array}{c}10 \\
(4-23)\end{array}$ & $\begin{array}{c}24 \\
(11-43)\end{array}$ & $\begin{array}{c}3 \\
(1-10)\end{array}$ & $\begin{array}{c}4 \\
(1-15)\end{array}$ & $\begin{array}{c}48 \\
(31-65) \\
\end{array}$ & $\begin{array}{c}12 \\
(5-26)^{c}\end{array}$ & $\begin{array}{c}4 \\
(1-15)\end{array}$ & $\begin{array}{c}8 \\
(2-24) \\
\end{array}$ & $\begin{array}{c}16 \\
(8-30) \\
\end{array}$ & $\begin{array}{c}27 \\
(15-44)\end{array}$ \\
\hline & & 2005-2009 & $\begin{array}{c}6 \\
(2-17) \\
\end{array}$ & $\begin{array}{c}9 \\
(4-17) \\
\end{array}$ & $\begin{array}{c}23 \\
(14-35) \\
\end{array}$ & $\begin{array}{c}1 \\
(0-4)\end{array}$ & $\begin{array}{c}0 \\
(0-1)\end{array}$ & $\begin{array}{c}49 \\
(37-62) \\
\end{array}$ & $\begin{array}{c}2 \\
(0-7)^{c}\end{array}$ & - & $\begin{array}{c}2 \\
(1-8) \\
\end{array}$ & $\begin{array}{c}24 \\
(15-38) \\
\end{array}$ & $\begin{array}{c}19 \\
(11-32)\end{array}$ \\
\hline \multirow{2}{*}{ All visits } & \multirow{2}{*}{63.4} & $2000-2004$ & - & $\begin{array}{c}9 \\
(5-16) \\
\end{array}$ & $\begin{array}{c}15 \\
(9-23) \\
\end{array}$ & $\begin{array}{c}3 \\
(1-7)\end{array}$ & $\begin{array}{c}9 \\
(5-15)^{c} \\
\end{array}$ & $\begin{array}{c}58 \\
(47-68) \\
\end{array}$ & $\begin{array}{c}7 \\
(4-13)^{\mathrm{d}} \\
\end{array}$ & $\begin{array}{c}1 \\
(0-4)\end{array}$ & $\begin{array}{c}8 \\
(4-16) \\
\end{array}$ & $\begin{array}{c}9 \\
(6-15) \\
\end{array}$ & $\begin{array}{c}18 \\
(12-27) \\
\end{array}$ \\
\hline & & 2005-2009 & $\begin{array}{c}4 \\
(2-8) \\
\end{array}$ & $\begin{array}{c}9 \\
(6-13) \\
\end{array}$ & $\begin{array}{c}12 \\
(9-17) \\
\end{array}$ & $\begin{array}{c}1 \\
(0-6) \\
\end{array}$ & $\begin{array}{c}2 \\
(1-5)^{c} \\
\end{array}$ & $\begin{array}{c}62 \\
(54-69) \\
\end{array}$ & $\begin{array}{c}1 \\
(0-2) \mathrm{d}\end{array}$ & - & $\begin{array}{c}4 \\
(2-9) \\
\end{array}$ & $\begin{array}{c}15 \\
(10-22) \\
\end{array}$ & $\begin{array}{c}13 \\
(9-18) \\
\end{array}$ \\
\hline
\end{tabular}

Note: empty cells indicate zero occurances in the sample

aRepresents patients prescribed at least 1 medication and nonpharmacologic treatment..

${ }^{b} P<0.01$.

${ }^{c} P<0.05$ for $2000-2004$ versus 2005-2009.

$d P<0.001$

nonpharmacologic monotherapy more frequently than gastroenterologists ( $\mathrm{OR}=3.9,95 \% \mathrm{CI}=1.2-12.2, \mathrm{P}=0.02)$.

\section{Opioid-Induced Constipation Alone}

Visits identified as relating to opioid-induced constipation totaled 3.7 million. Across all visits, there were no significant changes in the prescribing of major treatment categories or specific agents nor were there any associations between treatment and other factors (Appendix D, available in online article).

For each type of constipation, including IBS-C and opioidinduced constipation, there were few visits with concurrent medications (e.g., anticholinergics), comorbidities, or procedures with potential to influence constipation (Appendix E, available in online article).

\section{Discussion}

Since this study was conducted, updated treatment guidelines have become available, which include recommendations for the use of newly available agents, but the general recommendations have not changed. ${ }^{16,17}$ In this study, the prevalence of constipation and possibly IBS-C is increasing when compared with previous findings, which may be related to an increasing elderly population who are more likely to have constipation than other age groups. ${ }^{13,18}$ Given that the first presentation of IBS is typically between the ages of 30 to 50 years, it would be expected that the prevalence increases as the older population increases. This increased health care demand for constipation services reinforces the need for more evidence-based prescribing in order to reach optimal health outcomes and, ultimately, increase the quality of life for these individuals.

The findings of a decrease in prescribing combination therapy for patients aged $<18$ years and an increase in prescribing medication monotherapy for patients of all ages with constipation (including IBS-C) is concerning, since this finding contradicts national recommendations for the treatment of constipation..$^{910}$ According to expert recommendations for the treatment of constipation, all children and adolescents should receive combination therapy, and all adults on 1 or more medications should also be on therapy with nonpharmacologic treatments. ${ }^{1,9,12}$ All patients on a medication for constipation should also be prescribed nonpharmacologic therapies.

For visits because of constipation not related to IBS-C, there was also a decrease in prescribing combination therapy for patients aged $<18$ years and an increase in medication only 
Prescribing Patterns for Outpatient Treatment of Constipation, Irritable Bowel Syndrome-Related

Constipation, and Opioid-Induced Constipation: A Retrospective Cross-Sectional Study

\begin{tabular}{|c|c|c|c|c|c|c|c|c|c|c|}
\hline TABL & $\begin{array}{l}\text { Treatment Dif } \\
2000-2009, \text { P }\end{array}$ & $\begin{array}{l}\text { ces in Time } \\
\text { tage of IBS }\end{array}$ & $\begin{array}{l}\text { riod } \\
\text { its }\end{array}$ & $\begin{array}{l}\text { Major } \\
\text { ge) }\end{array}$ & eatn & Cate & & & & \\
\hline $\begin{array}{l}\text { Age Range, } \\
\text { Years }\end{array}$ & $\begin{array}{l}\text { Weighted } \\
\text { Number of Visits } \\
\text { (Millions) }\end{array}$ & Time Period & $\begin{array}{r}\text { Med } \\
\text { Nonph } \\
\mathrm{T} \\
\end{array}$ & $\begin{array}{l}\text { ion and } \\
\text { nacologic } \\
\text { apy }^{\mathrm{a}}\end{array}$ & Medic & on Only & \multicolumn{2}{|c|}{$\begin{array}{c}\text { Nonpharmacologic } \\
\text { Therapy Only }\end{array}$} & \multicolumn{2}{|c|}{ No Therapy } \\
\hline \multirow{2}{*}{$<18$} & \multirow{2}{*}{1.2} & $2000-2004$ & \multicolumn{2}{|c|}{-} & \multicolumn{2}{|c|}{-} & 28 & (11-55) & 72 & $(45-89)$ \\
\hline & & $2005-2009$ & 10 & $(2-37)$ & 8 & $(1-32)$ & 28 & $(11-54)$ & 54 & $(29-78)$ \\
\hline \multirow{2}{*}{$18-44$} & \multirow{2}{*}{9.4} & $2000-2004$ & 9 & $(4-18)$ & 4 & $(1-13)$ & 36 & $(26-47)^{\mathrm{b}}$ & 51 & $(40-62)$ \\
\hline & & $2005-2009$ & 5 & $(1-18)$ & 10 & $(4-21)$ & 19 & $(11-30)^{\mathrm{b}}$ & 67 & $(53-79)$ \\
\hline \multirow{2}{*}{$45-64$} & \multirow{2}{*}{10.5} & $2000-2004$ & 10 & $(5-19)^{b}$ & 4 & $(2-10)$ & 23 & $(15-34)$ & 63 & $(52-73)$ \\
\hline & & $2005-2009$ & 2 & $(1-6)^{b}$ & 12 & $(6-21)$ & 22 & $(11-40)$ & 64 & $(50-76)$ \\
\hline \multirow{2}{*}{$\geq 65$} & \multirow[b]{2}{*}{2.8} & $2000-2004$ & 9 & $(3-22)$ & 12 & $(5-27)$ & 18 & $(10-28)$ & 61 & $(47-74)$ \\
\hline & & $2005-2009$ & 7 & $(2-22)$ & 5 & $(2-10)$ & 26 & $(15-41)$ & 62 & $(48-74)$ \\
\hline \multirow{2}{*}{ All visits } & \multirow{2}{*}{28.2} & $2000-2004$ & 9 & $(6-14)$ & 6 & $(3-10)$ & 27 & (21-33) & 58 & $(52-65)$ \\
\hline & & 2005-2009 & 5 & $(2-10)$ & 9 & $(6-13)$ & 23 & $(16-31)$ & 64 & $(55-71)$ \\
\hline \multicolumn{11}{|c|}{$\begin{array}{l}\text { aRepresents patients prescribed at least } 1 \text { medication. } \\
b P<0.05 \text { for 2000-2004 versus 2005-2009. } \\
\text { IBS=irritable bowel syndrome. }\end{array}$} \\
\hline
\end{tabular}

for patients of all ages. In addition, there was a decrease in combination therapy for patients of any age and an increase in medication only in patients aged 45-64 years. Again, these findings for treating constipation are not consistent with the national recommendations for treatment. ${ }^{9,10}$ Considering specific choice of treatment, there were decreases in saline and lubricant use across all visits. Compared to previous findings, the current findings of an increase in medication only and a decrease in saline agents across all visits for constipation were consistent. ${ }^{12}$ Previously, the use of nonpharmacologic therapies was found to have decreased significantly over time, ${ }^{12}$ which is in contrast with the current findings of nonsignificant decreases in nonpharmacologic therapy only (21\% vs. 20\%). Furthermore, the percentage of visits resulting in a hyperosmolar was much higher in this study compared with a previous study (9\%-24\% vs. 58\%-62\%). ${ }^{13}$ The difference in hyperosmolar prescribing may be the result of heightened awareness of the safety, efficacy, and tolerability of polyethylene glycol. ${ }^{9,10}$

When comparing the different age groups, there were significant differences in the prescribing patterns for constipation. Although the elderly were not more likely to receive nonpharmacologic or pharmacologic treatment only for constipation, they were less likely to receive combination therapy compared with the other age groups. This could be an attempt to keep the complexity of the treatment regimen simple.

As expected, the percentage of females with constipation was greater than males ${ }^{9,10}$; however, females were less likely to receive combination therapy and more likely to receive nonpharmacologic therapy only. This may be because males are less likely to seek medical attention, and those that present with constipation do so only after their symptoms become severe, warranting initiation with combination therapy, or they already failed nonpharmacologic monotherapy and require adjunctive medication therapy.
Another interesting finding is that African-American patients were more likely to receive prokinetics than Caucasian patients. Although this study did not assess concurrent diabetes, the disproportionately higher prevalence of type 2 diabetes in African-Americans patients, ${ }^{15}$ and its potential complication of gastroparesis, may warrant greater use of prokinetics in this group in order to target the motility disorder.

For the prescribing patterns of IBS-C and opioid-induced constipation visits, there were no apparent deviations from the national recommendations. ${ }^{9,10}$ Interestingly, howerver, there was a significant increase in the prescription of tegaserod for IBS-C in patients aged 45-64 years, despite its change in status to a restricted drug in 2007, which limited its availability and the ability to prescribe it. Perhaps the increase in prescribing can be attributed to heightened awareness through U.S. Food and Drug Administration (FDA) announcements concerning the efficacy of the drug for patients that are refractory to other treatments. Females were also more likely to receive tegaserod for IBS-C than males, probably because of its product labeling. ${ }^{18}$

While the results of opioid-induced constipation were nonsignificant, the decreased use of stimulants and hyperosmolars are interesting, given that stimulants are recommended, and adjunctive treatment with hyperosmolars is also often recommended in this setting. Stimulants are preferred for opioidinduced constipation treatment and prevention because they specifically target the decreased peristalsis resulting from opioid use. Hyperosmolars are often used because they target the increased water absorption from the small and large colon. A newer drug, lubiprostone, may be an alternative because it facilitates passage of stool secondary to increased intestinal fluid secretions; however, there were no instances of lubiprostone use for opioid-induced constipation in this study. Interestingly, the manufacturer of lubiprostone has applied 


\section{Prescribing Patterns for Outpatient Treatment of Constipation, Irritable Bowel Syndrome-Related Constipation, and Opioid-Induced Constipation: A Retrospective Cross-Sectional Study}

\begin{tabular}{|c|c|c|c|c|c|c|c|c|c|c|c|}
\hline \multicolumn{2}{|c|}{ TABLE 7} & \multicolumn{10}{|c|}{$\begin{array}{l}\text { Treatment Differences in Time Periods by Medication Type, 2000-2009, } \\
\text { Percentage of IBS Visits with Prescribed Medication (Range) }\end{array}$} \\
\hline 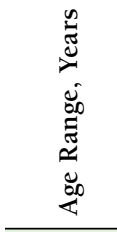 & 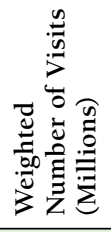 & 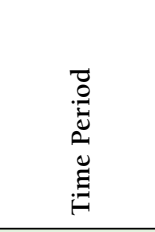 & 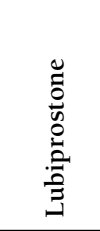 & 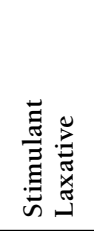 & 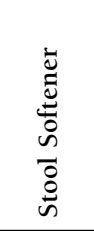 & 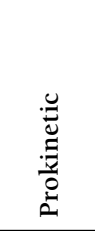 & 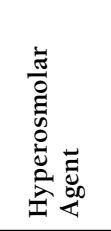 & 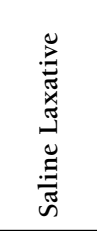 & 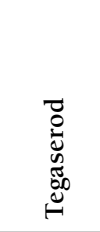 & 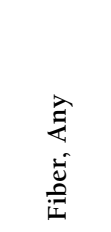 & 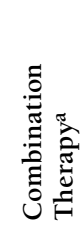 \\
\hline \multirow[b]{2}{*}{$<18$} & \multirow[b]{2}{*}{1.2} & $2000-2004$ & - & - & - & - & - & - & - & - & - \\
\hline & & 2005-2009 & - & $\begin{array}{c}37 \\
(4-88)\end{array}$ & - & - & $\begin{array}{l}100 \\
\text { (NA) }\end{array}$ & - & - & - & $\begin{array}{c}37 \\
(4-88) \\
\end{array}$ \\
\hline \multirow{2}{*}{$18-44$} & \multirow{2}{*}{9.4} & $2000-2004$ & - & $\begin{array}{c}3 \\
(0-17) \\
\end{array}$ & $\begin{array}{c}16 \\
(2-60)\end{array}$ & $\begin{array}{c}16 \\
(2-60)\end{array}$ & $\begin{array}{c}29 \\
(9-63)\end{array}$ & - & $\begin{array}{c}10 \\
(2-43)\end{array}$ & $\begin{array}{c}43 \\
(18-71)\end{array}$ & $\begin{array}{c}16 \\
(2-60) \\
\end{array}$ \\
\hline & & 2005-2009 & $\begin{array}{c}25 \\
(6-64) \\
\end{array}$ & - & - & $\begin{array}{c}10 \\
(2-36)\end{array}$ & $\begin{array}{c}5 \\
(1-33) \\
\end{array}$ & - & $\begin{array}{c}31 \\
(9-68) \\
\end{array}$ & $\begin{array}{c}34 \\
(9-73) \\
\end{array}$ & $\begin{array}{c}5 \\
(1-33)\end{array}$ \\
\hline \multirow{2}{*}{$45-64$} & \multirow{2}{*}{10.5} & $2000-2004$ & - & $\begin{array}{c}4 \\
(1-27) \\
\end{array}$ & $\begin{array}{c}7 \\
(1-38) \\
\end{array}$ & $\begin{array}{c}19 \\
(6-46) \\
\end{array}$ & $\begin{array}{c}36 \\
(14-66) \\
\end{array}$ & - & $\begin{array}{c}6 \\
(1-33)^{\mathrm{b}} \\
\end{array}$ & $\begin{array}{c}27 \\
(10-57) \\
\end{array}$ & - \\
\hline & & 2005-2009 & - & - & - & $\begin{array}{c}14 \\
(3-51) \\
\end{array}$ & $\begin{array}{c}47 \\
(20-77) \\
\end{array}$ & - & $\begin{array}{c}27 \\
(9-59)^{b}\end{array}$ & $\begin{array}{c}12 \\
(3-34) \\
\end{array}$ & - \\
\hline \multirow{2}{*}{$\geq 65$} & \multirow{2}{*}{2.8} & $2000-2004$ & - & - & $\begin{array}{c}5 \\
(1-22) \\
\end{array}$ & - & - & $\begin{array}{c}8 \\
(1-40) \\
\end{array}$ & $\begin{array}{c}44 \\
(17-75) \\
\end{array}$ & $\begin{array}{c}43 \\
(17-74) \\
\end{array}$ & - \\
\hline & & 2005-2009 & - & $\begin{array}{c}4 \\
(0-27) \\
\end{array}$ & - & $\begin{array}{c}35 \\
(7-79) \\
\end{array}$ & $\begin{array}{c}14 \\
(3-46) \\
\end{array}$ & - & $\begin{array}{c}5 \\
(1-32) \\
\end{array}$ & $\begin{array}{c}73 \\
(38-92) \\
\end{array}$ & - \\
\hline \multirow{2}{*}{ All visits } & \multirow{2}{*}{28.2} & $2000-2004$ & - & $\begin{array}{c}2 \\
(1-10) \\
\end{array}$ & $\begin{array}{c}9 \\
(3-28) \\
\end{array}$ & $\begin{array}{c}12 \\
(3-36)\end{array}$ & $\begin{array}{c}23 \\
(11-42)\end{array}$ & $\begin{array}{c}2 \\
(0-15)\end{array}$ & $\begin{array}{c}19 \\
(8-37)\end{array}$ & $\begin{array}{c}37 \\
(22-56)\end{array}$ & $\begin{array}{c}5 \\
(1-29)\end{array}$ \\
\hline & & 2005-2009 & $\begin{array}{c}8 \\
(2-27) \\
\end{array}$ & $\begin{array}{c}3 \\
(1-14) \\
\end{array}$ & - & $\begin{array}{c}17 \\
(6-39) \\
\end{array}$ & $\begin{array}{c}29 \\
(15-50) \\
\end{array}$ & - & $\begin{array}{c}21 \\
(10-39) \\
\end{array}$ & $\begin{array}{c}33 \\
(17-55) \\
\end{array}$ & $\begin{array}{c}4 \\
(1-16) \\
\end{array}$ \\
\hline
\end{tabular}

for an FDA indication for opioid-induced constipation; thus, lubiprostone may become a more common treatment for this indication in the future.

Our results show that there is little consistency in prescribing patterns for the different types of constipation, which points to a lack of evidence-based prescribing. The lack of evidence-based prescribing may compromise optimal symptom improvement. It may also impact payers by increasing the demand for physician visits and, thus, increase health care utilization and costs related to constipation. Streamlining treatments based on the available, albeit limited, evidence may lead to enhanced patient satisfaction and improved outcomes. Health plans may benefit from this streamlined approach not just by improving the patient experience and outcomes, but also by reducing health care utilization and costs associated with constipation.

\section{Limitations}

Because of the retrospective study methodology, the data available were limited to the fields available, and accuracy was dependent on each physician who entered data. The results are representative of data from a finite period, preventing assessment of the course of disease and treatment, and it was not possible to determine what treatment options were tried or discussed at past visits. Further, the sample size for opioidinduced constipation and IBS-C visits were small, limiting the findings of significance.

While the NAMCS database includes information on the prescription of over-the-counter medications, patients self-treating with these agents are not captured, limiting the generalizability of the results. This is of particular importance, given that the majority of the agents used to treat constipation are available over the counter. The constipation treatments available at the time of this study that were available by prescription only were anthraquinone- or diphenylmethane-containing products, lactulose, sorbitol, misoprostol, lubiprostone, tegaserod, botulinum toxin, cisapride, and metoclopramide. In addition, changes in availability and regulations of lubiprostone and tegaserod from 2000 to 2009 likely influenced prescribing patterns.

\section{Conclusions}

Health care utilization resulting from constipation increased and the prescribing patterns for treatment have shifted significantly from 2000 to 2009 for constipation and IBS-C. The treatment patterns were significantly influenced by age, 


\section{Prescribing Patterns for Outpatient Treatment of Constipation, Irritable Bowel Syndrome-Related Constipation, and Opioid-Induced Constipation: A Retrospective Cross-Sectional Study}

gender, race, ethnicity payment source, physician specialty, and region across all ages and within age groups. Overall changes in treatment categories over time included a decrease in combination therapy for patients aged $<18$ years and an increase in medication monotherapy across all ages, which are in contrast to the national recommendations. The implications of the identified patterns may include increased health care utilization and lead to additional costs to payers. Accessing constipation-prescribing patterns within their own plan populations may offer payers an opportunity to optimize treatment outcomes and costs.

\section{Authors}

KATY E. TRINKLEY, PharmD, is Assistant Professor, Department of Clinical Pharmacy, University of Colorado Skaggs School of Pharmacy and Pharmaceutical Sciences, Aurora; BRUCE E. SILL, PharmD, MS, is Director, Health Economics \& Outcomes Research, Bristol-Myers Squibb, Princeton, New Jersey; and KYLE PORTER, MAS, is Senior Biostatistician, Center for Biostatistics, The Ohio State University, Columbus. MILAP C. NAHATA, PharmD, MS, is Director, Institute of Therapeutic Innovations and Outcomes, and Professor Emeritus, Pharmacy, Pediatrics and Internal Medicine, Colleges of Pharmacy and Medicine, The Ohio State University and Nationwide Children's Hospital and Research Institute, Columbus, Ohio.

AUTHOR CORRESPONDENCE: Milap C. Nahata, PharmD, MS, Director, Institute of Therapeutic Innovations and Outcomes, and Professor Emeritus, Pharmacy, Pediatrics and Internal Medicine, Colleges of Pharmacy and Medicine, The Ohio State University, 500 W. 12th Ave., Columbus, OH 43210. Tel.: 614.292.2472;

Fax:614.292.2588; E-mail: nahata.1@osu.edu.

\section{REFERENCES}

1. American College of Gastroenterology Task Force on Irritable Bowel Syndrome. An evidence-based position statement on the management of irritable bowel syndrome in North America. Am J Gastroenterol. 2002 97(11 Suppl):S1-5.

2. Wald A, Scarpignato C, Kamm MA, et al. The burden of constipation on quality of life: results of a multinational survey. Aliment Pharmacol Ther. 2007;26(2):227-36.

3. Sonnenberg A, Koch TR. Physician visits in the United States for constipation: 1958 to 1986. Dig Dis Sci. 1989;34(4):606-11.

4. Loening-Baucke V. Prevalence rates for constipation and faecal and urinary incontinence. Arch Dis Child. 2007;92(6):486-89.

5. Martin BC, Barghout V, Cerulli A. Direct medical costs of constipation in the United States. Manag Care Interface. 2006;19(12):43-49.

6. Eoff JC, Lembo AJ. Optimal treatment of chronic constipation in managed care: a review and roundtable discussion. J Manag Care Pharm. 2008; 14(9 Suppl A):1-15. Available at: http://www.amcp.org/data/jmcp/ November\%202008\%20supplement.pdf.

7. American College of Gastroenterology Chronic Constipation Task Force. An evidence-based approach to the management of chronic constipation in North America. Am J Gastroenterol. 2005;100(Suppl 1):S1-4.

8. Ford AC, Suares NC. Effect of laxatives and pharmacological therapies in chronic idiopathic constipation: systematic review and meta-analysis. Gut. 2011;60(2):209-18.

9. Constipation Guideline Committee of the North American Society for Pediatric Gastroenterology, Hepatology and Nutrition. Evaluation and treatment of constipation in infants and children: recommendations of the North American Society for Pediatric Gastroenterology, Hepatology and Nutrition. J Pediatr Gastroenterol Nutr. 2006;43(3):el-13.

10. Locke GR 3rd, Pemberton JH, Phillips SF. AGA technical review on constipation. American Gastroenterological Association. Gastroenterology. 2000;119(6):1766-78.

11. Woolery M, Bisanz A, Lyons HF, et al. Putting evidence into practice: evidence-based interventions for the prevention and management of constipation in patients with cancer. Clin J Oncol Nurs. 2008;12(12):317-37.

12. Trinkley KE, Porter K, Nahata MC. Prescribing patterns for the outpatient treatment of constipation in the U.S. Dig Dis Sci. 2010;55(12):3514-20

13. Centers for Disease Control and Prevention. Ambulatory health care data. Available at: http://www.cdc.gov/nchs/about/major/ahcd/ahcdl.htm. Accessed October 6, 2015

14. Woelk CJ. The hand that writes the opioid... Can Fam Physician. 2007;53(6):1015-17

15. Mayer-Davis EJ, Beyer J, Bell RA, et al. Diabetes in African American youth: prevalence, incidence, and clinical characteristics: the SEARCH for Diabetes in Youth Study. Diabetes Care. 2009;32(Suppl 2):S112-22.

16. Bharucha AE, Pemberton JH, Locke GR 3rd. American Gastroenterological Association technical review on constipation. Gastroenterology. 2013;144(1):218-38.

17. American College of Gastroenterology Task Force on Irritable Bowel Syndrome, Brandt LJ, Chey WD, et al. An evidence-based position statement on the management of irritable bowel syndrome. Am J Gastroenterol. 2009;104(Suppl 1):S1-35

18. Sandler RS. Epidemiology of irritable bowel syndrome in the United States. Gastroenterology. 1990;99(2):409-15. 


\section{Prescribing Patterns for Outpatient Treatment of Constipation, Irritable Bowel Syndrome-Related Constipation, and Opioid-Induced Constipation: A Retrospective Cross-Sectional Study}

\begin{tabular}{l|l}
\hline \multicolumn{1}{c}{ APPENDIX A } & $\begin{array}{l}\text { List of ICD-9-CM Codes for the } \\
\text { Diagnosis of Constipation }\end{array}$ \\
\hline ICD-9-CM Code & \multicolumn{1}{c}{ Diagnosis } \\
\hline 564.0 & Constipation \\
\hline 564.09 & Other constipation \\
\hline 564.00 & Constipation, unspecified \\
\hline 564.02 & Outlet dysfunction constipation \\
\hline 564.01 & Slow transit constipation \\
\hline 564.1 & Irritable bowel syndrome \\
\hline $\begin{array}{l}\text { ICD-9-CM = International Classification of Diseases, Ninth Revision, Clinical } \\
\text { Modification. }\end{array}$
\end{tabular}

\section{APPENDIX B Medications Used for Constipation and NAMCS Medication Codes}

\begin{tabular}{|c|c|c|}
\hline Medications & Type & NAMCS Medication Codes \\
\hline Anthraquinones-senna & \multirow{4}{*}{ Stimulant laxative } & 55200,04615 \\
\hline Anthraquinones-cascara, casanthranol & & 50935 \\
\hline Phenolphthalein & & 54405 \\
\hline Diphenylmethane- bisacodyl & & 50630,04370 \\
\hline Docusate sodium & Stool softener & 51935 \\
\hline Mineral oil & Lubricant & 17660,53720 \\
\hline Polyethylene glycol & \multirow{4}{*}{ Hyperosmolar agent } & $54590,94029,42425,03437$ \\
\hline Lactulose & & 52995 \\
\hline Sorbitol & & 28915,55435 \\
\hline Glycerin suppository & & $12622,27429,22145,98059$ \\
\hline Mg hydroxide & Saline laxative & $19375,24058,41865$ \\
\hline Sodium phosphate & Bowel evacuant & 55385 \\
\hline Castor oil & \multirow{6}{*}{ Miscellaneous } & 25749,05885 \\
\hline Colchicine & & 51345 \\
\hline Misoprostol & & 91011 \\
\hline Lubiprostone & & 71035 \\
\hline Tegaserod & & 70642 \\
\hline Botulinum toxin & & 702166,70502 \\
\hline Cisapride & \multirow{2}{*}{ Prokinetic } & 56395 \\
\hline Metoclopramide & & 53688 \\
\hline Calcium polycarbophil & \multirow{3}{*}{ Fiber } & 56715,54587 \\
\hline Psyllium & & 54965 \\
\hline Methylcellulose & & $06020,07345,19140,34255,60325$ \\
\hline Stimulant laxative/stool softener & Combination stimulant/stool softener & $04176,04059,27795,07268,09945$ \\
\hline Stimulant/fiber & Combination stimulant/fiber & $27805,23415,05890$ \\
\hline Homeopathic & Combination homeopathic product & $04213,17460,04147$ \\
\hline Miscellaneous combinations & Combination product, other & $\begin{array}{l}13330,25449,27755,35020,05865,04283,05830,05835,05865, \\
12588,19380,16790,22260,20410,33850,30625,33855,16795, \\
17245,27480,41270,61175,40630,10090,40635,60475,19385, \\
14950,30300,09715\end{array}$ \\
\hline Barium bisacodyl enema & Enema & 96074 \\
\hline
\end{tabular}




\section{APPENDIX C Surgeries/Procedures for Treatment or Diagnosis of Constipation and ICD-9-CM Codes}

\begin{tabular}{l|l}
\hline ICD-9-CM Code & \multicolumn{1}{c}{ Surgery/Procedure Therapy } \\
\hline 96.29 & $\begin{array}{l}\text { Reduction of intussusception of alimentary tract } \\
\text { With: fluoroscopy, ionizing radiation enema, ultrasonography guidance, hydrostatic reduction, pneumatic reduction }\end{array}$ \\
\hline 46.80 & $\begin{array}{l}\text { Intra-abdominal manipulation of intestine, not otherwise specified } \\
\text { Correction of intestinal malrotation } \\
\text { Reduction of: intestinal torsion, intestinal volvulus, intussusception }\end{array}$ \\
\hline 45.8 & $\begin{array}{l}\text { Total intra-abdominal colectomy } \\
\text { Excision of cecum, colon, and sigmoid }\end{array}$ \\
\hline 46.92 & Myotomy of other parts of colon \\
\hline 94.39 & Other individual psychotherapy: biofeedback \\
\hline 45.24 & $\begin{array}{l}\text { Flexible sigmoidoscopy } \\
\text { Endoscopy of descending colon }\end{array}$ \\
\hline 96.39 & Other transanal enema \\
\hline 45.23 & Colonoscopy \\
\hline 46.8 & Dilation and manipulation of intestine \\
\hline ICD-9-CM=International Classification of Diseases, Ninth Revision, Clinical Modification.
\end{tabular}

APPENDIX D Treatment Differences in Time Periods by Major Treatment Category, 2000-2009, Percentage of Opioid-Induced Constipation Visits (Range)

\begin{tabular}{|c|c|c|c|c|c|c|c|c|c|}
\hline Age Range, Years & $\begin{array}{c}\text { Weighted } \\
\text { Number of Visits } \\
\text { (Millions) }\end{array}$ & Time Period & $\begin{array}{c}\text { Medication and } \\
\text { Nonpharmacologic } \\
\text { Therapy }\end{array}$ & Med & tion Only & $\begin{array}{c}\text { Nonpl } \\
\text { The }\end{array}$ & $\begin{array}{l}\text { nacologic } \\
\text { y Only }\end{array}$ & No & herapy \\
\hline \multirow{2}{*}{$18-44$} & \multirow{2}{*}{0.6} & $2000-2004$ & - & & - & 37 & $(7-83)$ & 63 & $(18-93)$ \\
\hline & & $2005-2009$ & - & 66 & $(19-94)$ & \multicolumn{2}{|c|}{-} & 34 & $(6-81)$ \\
\hline \multirow{2}{*}{$45-64$} & \multirow{2}{*}{1.3} & $2000-2004$ & $(14-74)$ & 12 & $(2-53)$ & 6 & $(1-35)$ & 42 & $(17-72)$ \\
\hline & & 2005-2009 & $(3-30)$ & 37 & $(13-71)$ & 3 & $(0-18)$ & 50 & $(21-79)$ \\
\hline \multirow{2}{*}{$\geq 65$} & \multirow{2}{*}{1.8} & $2000-2004$ & - & 21 & $(3-69)$ & 11 & $(1-50)$ & 68 & $(28-92)$ \\
\hline & & 2005-2009 & - & 33 & $(14-60)$ & 24 & $(8-53)$ & 43 & $(22-68)$ \\
\hline \multirow{2}{*}{ All visits } & \multirow{2}{*}{3.7} & $2000-2004$ & $(5-37)$ & 14 & $(3-45)$ & 13 & $(4-34)$ & 58 & $(36-78)$ \\
\hline & & $2005-2009$ & $(1-10)$ & 39 & $(25-56)$ & 13 & $(5-30)$ & 45 & $(28-63)$ \\
\hline
\end{tabular}

Note: empty cells indicate zero occurances in the sample.

aRepresents patients prescribed at least 1 medication.

APPENDIX E Weighted Percentage of Visits Associated with at Least 1 Concurrent Medication, Comorbidity, Treatment Procedure, and Diagnostic Procedure (Range)

\begin{tabular}{|c|c|c|c|c|c|c|c|c|}
\hline \multirow[b]{2}{*}{ Concurrent medication } & \multicolumn{2}{|c|}{$\begin{array}{l}\text { Any Constipation } \\
\text { and/or IBS } \\
\text { (Group 1) }\end{array}$} & \multicolumn{2}{|c|}{$\begin{array}{c}\text { Constipation, Excludes IBS } \\
\text { Without Constipation } \\
\text { (Group 2) }\end{array}$} & \multicolumn{2}{|c|}{$\begin{array}{c}\text { IBS } \\
\text { (Group 3) }\end{array}$} & \multicolumn{2}{|c|}{$\begin{array}{c}\text { Opioid-Induced } \\
\text { Constipation }\end{array}$} \\
\hline & 5.7 & $(4.5-7.3)$ & 5.7 & $(4.2-7.5)$ & 6.1 & $(4.4-8.6)$ & 100.0 & \\
\hline Comorbidity & 13.3 & $(11.4-15.6)$ & 13.0 & $(10.8-15.7)$ & 13.4 & $(10.2-17.4)$ & 14.6 & $(6.8-28.6)$ \\
\hline Procedure, treatment & 0.0 & & 0.0 & & 0.0 & & 0.0 & \\
\hline Diagnostic procedure & 10.6 & $(8.1-13.9)$ & 11.7 & $(8.8-15.4)$ & 8.4 & $(5.5-12.8)$ & 5.8 & $(1.8-16.7)$ \\
\hline
\end{tabular}

